\title{
a-Glucosidase Inhibitory Activity in Rice Miso Supplementary with Black Soybean
}

\author{
Chengyu Jiang ${ }^{1,2}$, Zhaohong $\mathrm{Ci}^{1,2}$, Michiyuki Kojima ${ }^{1,2, *}$ \\ ${ }^{1}$ Department of Food Science, Obihiro University of Agriculture and Veterinary Medicine, 080-8555, \\ 11, Nishi-2-Sen, Inada-Cho, Obihiro, Hokkaido, Japan \\ ${ }^{2}$ Department of Bioresources Science, United Graduate School of Agricultural Sciences, Iwate University, 020-8550, \\ 3-18-8, Ueda, Morioka, Iwate, Japan \\ *Corresponding author: kojima@obihiro.ac.jp
}

Received December 06, 2018; Revised January 10, 2019; Accepted February 06, 2019

\begin{abstract}
In the present study, we processed rice miso supplementary with black soybean (RM-BS), and analyzed $\alpha$-glucosidase inhibitory activity, polyphenol and melanoidin content at different fermentation periods (3, 6, 24, 36 months). The results showed $\alpha$-glucosidase inhibitory activity, polyphenol and melanoidin content in RM-BS were increased with prolonging the fermentation periods. The $\alpha$-glucosidase inhibitory activity in RM-BS was significantly stronger at different fermentation periods than those of RM, respectively. There were high positive relationships between $\alpha$-glucosidase inhibitory activity and polyphenol content, and melanoidin content, respectively. Moreover, the ratio of melanoidin and polyphenol content was increased with prolonging the fermentation periods. So thus, we speculated that melanoidins were the main $\alpha$-glucosidase inhibitory activity component in RM-BS. These results could be useful on researching and developing of rice miso products.
\end{abstract}

Keywords: rice miso, black soybean, $\alpha$-glucosidase inhibitory activity, polyphenol, melanoidin

Cite This Article: Chengyu Jiang, Zhaohong Ci, and Michiyuki Kojima, " $\alpha$-Glucosidase Inhibitory Activity in Rice Miso Supplementary with Black Soybean.” American Journal of Food Science and Technology, vol. 7, no. 1 (2019): 27-30. doi: 10.12691/ajfst-7-1-5.

\section{Introduction}

In recent years, dietary life of people is more inclined to meat [1], dairy products [2], vegetable oils, tobacco, sugary foods, Coca-Cola, and alcoholic beverages. These unhealthy dietary habits are at high risk of developing lifestyle diseases, such as diabetes, obesity, cancer, and metabolic syndrome [3,4,5,6] for middle aged and elderly people. Moreover, more and more young people are suffering from these diseases [7].

Soybeans and their fermented products such as touchi, natto, tempeh played significant roles in the Asian diets $[8,9,10]$. As a traditional fermentation food in Japan, rice miso demonstrated some beneficial effects, such as lipid peroxidation-inhibiting action, anti-hypertension, anti- mutagen action [11,12].

In the Compendium of Materia Medica, black soybeans were used as a traditional Chinese medicine. Yoshida, 2013 [13] reported that black soybeans have blood-activating, detoxifying and diuresis-promoting. Moreover, black soybean contained a large amount of dietary fiber, flavonoid and anthocyanin, and these components have with strong antioxidant activity, glycolysis-inhibitory activity, anti-obesity, anti-cancer and hepato-protective effect $[14,15,16,17,18]$.

In this research, we processed rice miso supplementary with black soybean (RM-BS), and detected $\alpha$-glucosidase inhibitory activity, polyphenol and melanoidin content at different fermentation periods.

\section{Materials and Methods}

\subsection{Materials and Chemicals}

Black soybean (Glycine max), soybean (Glycine max), rice-malt, salt, and seed miso were purchased from supermarket (Obihiro, Japan). Rice-malt was purchased from the Salt Industry Center (Tokyo, Japan). The rice miso products were manufactured and sampled for analysis at 3, 6, 24, 36 Months (M) of fermentation.

Folin-Ciocalteau reagent was obtained from Nacalai Tesque, Inc. (Kyoto, Japan). DNS (3,5-dinitrosalicylic acid) were purchased from Sigma-Aldrich Co., LLC. (Tokyo, Japan). Glucose and glycine were purchased from Kanto Chemical Co., Inc. (Tokyo, Japan). The other reagents were purchased from Wako Pure Chemical Industries, Ltd. (Osaka, Japan).

\subsection{Extraction and Fractionation}

The RM-BS was manufactured by industrial producing rice miso and extracted by $80 \%$ ethanol and $70 \%$ acetone [19]. The extracts of RM-BS were concentrated and dissolved by distilled water. The distilled water fraction was delaminated by n-hexane and ethyl acetate. Then, the water layer was fractioned by HP-20 column. After HP-20 
column, the methanol fractions were analyzed as the following experiments.

\section{3. $\alpha$-Glucosidase Inhibitory Activity Assay}

$\alpha$-Glucosidase inhibitory activity was determined by the improved DNS method [15]. A mixture of $0.5 \mathrm{~mL}$ of sample extract with different concentration $(0.05,0.1,0.2$ g) and $0.5 \mathrm{~mL}$ of the $\alpha$-glucosidase solution was pre- incubated at $37^{\circ} \mathrm{C}$ for 10 min to prepare solution I. A mixture of $50 \mu \mathrm{L}$ of $0.4 \%$ sucrose solution, $625 \mu \mathrm{L}$ of $0.1 \mathrm{M} \mathrm{Na}_{2} \mathrm{PO}_{4}$ buffer (pH 6.8) and $125 \mu \mathrm{L}$ of $1 \% \mathrm{NaCl}$ was pre-incubated at $37^{\circ} \mathrm{C}$ for $10 \mathrm{~min}$ to prepare solution II. Then, $200 \mu \mathrm{L}$ of solution I was mixed with solution II and incubated at $37^{\circ} \mathrm{C}$ for $30 \mathrm{~min}$. The enzyme reaction was stopped by adding $125 \mu \mathrm{L}$ of $2 \mathrm{~N} \mathrm{NaOH}$ (added $2 \mathrm{~N}$ $\mathrm{NaOH}$ before incubation for blank). DNS solvent (1\%, $125 \mu \mathrm{L}$ ) was added and reacted in boiling water bath for $10 \mathrm{~min}$. Absorbance was measured at $540 \mathrm{~nm}$. Standard curves were using glucose calibration, and expressed as $\alpha$-glucosidase inhibitory activity (\%).

$$
\begin{aligned}
\alpha & - \text { Glucosidase inhibitory activity (\%) } \\
= & {\left[1-\left(\frac{\mathrm{A}_{\text {sample }}-\mathrm{A}_{\text {blank }}}{\mathrm{A}_{\text {control }}-\mathrm{A}_{\text {test }}}\right)\right] \times 100 \% }
\end{aligned}
$$

where $A_{\text {sample }}$ is the absorbance of the mixture of sample, sucrose solution, enzyme and DNS solvent; $A_{\text {blank }}$ is the absorbance of the mixture of sample, sucrose solution and DNS solvent without enzyme; $A_{\text {control }}$ is the absorbance of the mixture of buffer (instead of sample), sucrose solution, enzyme and DNS solvent; $A_{\text {test }}$ is the absorbance of the mixture of buffer (instead of sample), sucrose solution and DNS solvent without enzyme.

\subsection{Polyphenol and Melanoidin Content Determination}

Polyphenol content was determined using the method of Folin-Ciocelteau [22] with slight modifications. Each sample $(100 \mu \mathrm{L})$ was mixed with $300 \mu \mathrm{L}$ of distilled water, $400 \mu \mathrm{L}$ of $50 \%$ Folin-Ciocelteu reagent, and $400 \mu \mathrm{L}$ of $10 \%$ $\mathrm{Na}_{2} \mathrm{CO}_{3}$ aqueous solution. The reaction solution was incubated at $30^{\circ} \mathrm{C}$ for $30 \mathrm{~min}$, and centrifuged at $1,006 \times \mathrm{g}$ for $10 \mathrm{~min}$. Thereafter, the absorbance was measured at $760 \mathrm{~nm}$, and results were expressed as the mg catechin per gram DW miso $\left(y=10.709 x-0.9949, r^{2}=0.9931\right)$.

Melanoidin content was determined by the method of Martins [23]. Firstly, glucose and glycine was mixed as melanoidin standard. Then, melanoidin standard with different concentration (0, 0.2, 0.4, 0.6, 0.8, $1.0 \mathrm{mg} / \mathrm{mL}$ ) and each sample $(100 \mu \mathrm{L})$ was mixed with $900 \mu \mathrm{L}$ of distilled water. The absorbance was measured at $450 \mathrm{~nm}$. The results were expressed as the mg melanoidin per gram DW miso $\left(\mathrm{y}=61.333 \mathrm{x}+1.0356, \mathrm{r}^{2}=0.9994\right)$.

\subsection{Statistical Analysis}

The experiments were repeated at least three times. Data were expressed as means \pm standard deviation. Significant differences were determined by one-way ANOVA and Fisher's test (SAS v. 7.1, SAS Institute Inc.,
Cary, NC, USA). Differences were considered to be significant at $P<0.05$.

\section{Results and discussion}

\section{1. $\alpha$-Glucosidase Inhibitory Activity in RM-BS}

$\alpha$-Glucosidase inhibitory activity of RM-BS and RM were shown in Figure 1. $\alpha$-Glucosidase inhibitory activity in RM-BS and RM were significantly increasing with prolonging fermentation period, and the strongest inhibitory activity was shown at 24 Months fermentation. Moreover, $\alpha$-glucosidase inhibitory activity at 3, 6, 24, 36 Months fermentation in RM-BS were significantly higher than those of RM (control; rice miso), respectively. $\alpha$-Glucosidase inhibitory activity in rice miso supplementary with kidney bean at different fermentation periods were also significantly higher than those of rice miso [24]. There are also reported that soybean paste, natto, and touchi have $\alpha$-glucosidase inhibitory activity $[25,26,27]$.

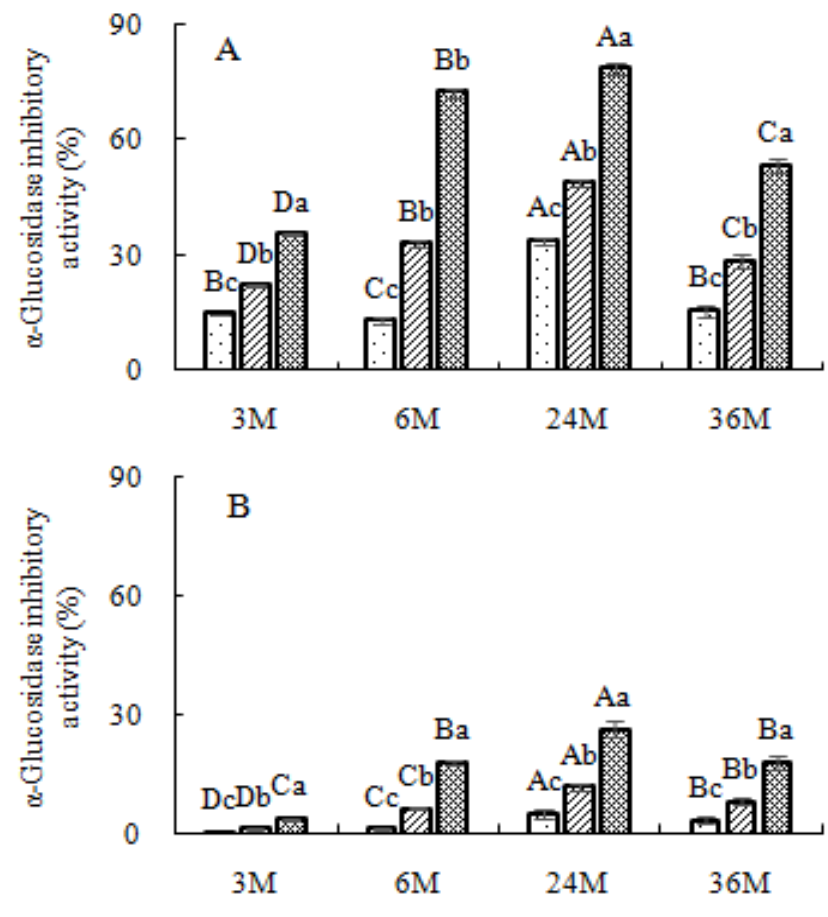

Figure 1. $\alpha$-Glucosidase inhibitory activity contained in rice miso products with different fermentation periods. A, RM-BS. B, RM. $\square$ for the $0.05 \mathrm{~g}$ DW miso; $\square$ for the $0.1 \mathrm{~g} \mathrm{DW}$ miso; $0.2 \mathrm{~g} \mathrm{DW}$ miso. Values within a column followed by different small letters and values within a row followed by different capital letters are significant at $p<0.05$.

\subsection{Polyphenol and Melanoidin Content in RM-BS}

The results of polyphenol and melanoidin content in RM-BS were shown in Table 1. Polyphenol content was increasing with prolonging the fermentation periods. The highest polyphenol value of RM-BS was shown at 36 Months fermentation ( $3.4 \mathrm{mg} / \mathrm{g} \mathrm{DW}$ miso). The polyphenol content of RM-BS was increased by 3.9 folds 
from 3 months to 36 months. There are similar results of total phenolic contents in soybean fermented products were increased with extension of fermentation $[28,29]$.

We found positive relationships between $\alpha$-glucosidase inhibitory activity and polyphenol content, and melanoidin content, respectively. The correlation coefficients were 0.8148 for former, and 0.7686 for latter, respectively (Figure 2). Glucose-amino acid model MRPs showed $\alpha$-glucosidase inhibitory activity [30]. Polyphenolic compounds are strong inhibitors of $\alpha$-glucosidase inhibitory activity, and serve as a potent alternative to avoid the adverse effects [31]. The ratio of melanoidin and polyphenol content was approximately 3:1 at 3 Months fermentation, and that of 6:1 at 36 Months fermentation. Melanoidin ratio was increased with the prolonging fermentation period. We speculated that melanoidins were the main $\alpha$-glucosidase inhibitory activity component rather than polyphenols in RM-BS.

Table 1. Polyphenol and melanoidin content in RM-BS with different fermentation periods

\begin{tabular}{ccc}
\hline Sample & $\begin{array}{c}\text { Polyphenol content } \\
\text { (mg/g DW miso) }\end{array}$ & $\begin{array}{c}\text { Melanoidin content } \\
\text { (mg/g DW miso) }\end{array}$ \\
\hline RM-BS-3M & $0.9 \pm 0.1 \mathrm{Bd}$ & $3.1 \pm 0.1 \mathrm{Ad}$ \\
RM-BS-6M & $2.1 \pm 0.3 \mathrm{Bc}$ & $11.4 \pm 0.5 \mathrm{Ac}$ \\
RM-BS-24M & $2.9 \pm 0.3 \mathrm{Bb}$ & $15.6 \pm 0.3 \mathrm{Ab}$ \\
RM-BS-36M & $3.4 \pm 0.4 \mathrm{Ba}$ & $19.9 \pm 0.8 \mathrm{Aa}$ \\
\hline
\end{tabular}

Abbreviations: M, month; DW, dry weight; RM-BS, rice miso supplementary with black soybean. Data represent the mean \pm SD from at least three independent studies. Values within a row followed by different capital letters and values within a column followed by different small letters are significant at $p<0.05$.
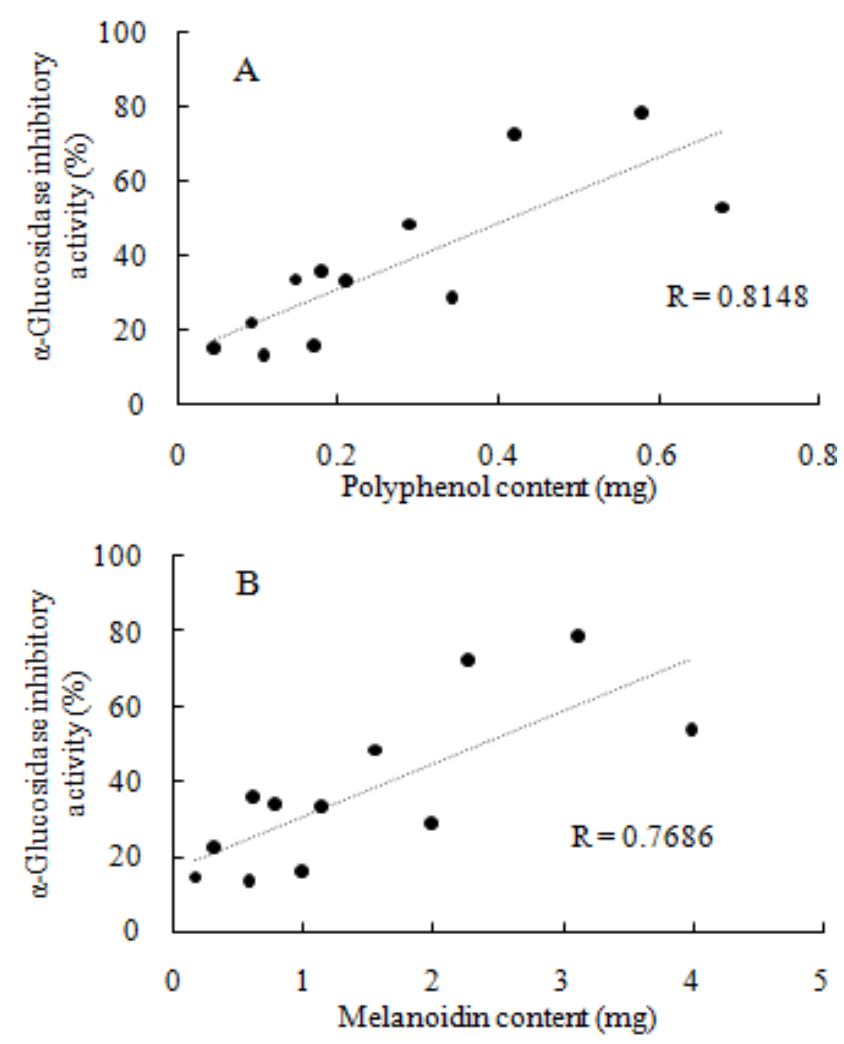

Figure 2. Relationship between $\alpha$-glucosidase inhibitory activity and polyphenol content, melanoidin content in RM-BS

\section{Conclusion}

In the present study, we detected $\alpha$-glucosidase inhibitory activity, polyphenol and melanoidin content in RM-BS were increased with prolonging fermentation periods. Moreover, the $\alpha$-glucosidase inhibitory activity in RM-BS was significantly higher than RM. There were high positive relationships between $\alpha$-glucosidase inhibitory activity and polyphenol content, and melanoidin content, respectively. Therefore, we speculated that the supplementation of black soybean in rice miso could improve $\alpha$-glucosidase inhibitory activity with prolonging fermentation periods. As comparing with polyphenols, melanoidins maybe the main component for $\alpha$-glucosidase inhibitory activity in RM-BS.

\section{Acknowledgments}

We acknowledge the financial support given by the Obihiro University of Agriculture and Veterinary Medicine, as well as Iwate University, for the completion of the study.

\section{Statement of Competing Interests}

The authors have no competing interests.

\section{List of Abbreviations}

M: month; RM: rice miso; RM-BS: rice miso supplementary with black soybean; DW: dry weight; DNS: 3, 5-dinitrosalicylic acid.

\section{References}

[1] Stokes, C., Peet, M., "Dietary sugar and polyunsaturated fatty acid consumption as predictors of severity of schizophrenia symptoms," Nutritional Neuroscience, 7(4). 247-249. Aug 2004.

[2] McCreadie, R.G., "Diet, smoking and cardiovascular risk in people with schizophrenia: descriptive study,” British Journal of Psychiatry, 183. 534-539. Dec 2003.

[3] Cimo, A., Stergiopoulos, E., Cheng, C., Bonato, S., Dewa, C.S., "Effective lifestyle interventions to improve type II diabetes self-management for those with schizophrenia or schizoaffective disorder: a systematic review," BMC Psychiatry, 12: 24. Mar 2012.

[4] Correll, C.U., "Prevalence, incidence and mortality from cardiovascular disease in patients with pooled and specific severe mental illness: a large-scale meta-analysis of 3,211,768 patients and 113,383,368 controls,” World Psychiatry, 16(2). 163-180. Jun 2017.

[5] Crump, C., Winkleby, M. A., Sundquist, K., Sundquist. J., "Comorbidities and mortality in persons with schizophrenia: A Swedish national cohort study," American Journal of Psychiatry, 170. 324-333. Mar 2013.

[6] Sundström, J., Risérus, U., Byberg, L., Zethelius, B., Lithell, H., Lind, L., "Clinical value of the metabolic syndrome for long term prediction of total and cardiovascular mortality: prospective, population based cohort study,” British Medical Journal, 332. 878-882. Apr 2006.

[7] World Health Organization, WHO Library Cataloguing- inPublication Data: Global report on diabetes, 2016. 
[8] Chen, J., Cheng, Y.Q., Yamaki, K., Li, L.T., "Anti-a-glucosidase activity of Chinese traditionally fermented soybean (douchi)," Food Chemistry, 103(4). 1091-1096. Dec 2007.

[9] Soka, S., Suwanto, A., Rusmana, I., Sajuthi, D., Iskandriati, D., Jessica, K., "Analysis of Intestinal Mucosal Immunoglobulin A in Sprague Dawley Rats Supplemented with Tempeh," HAYATI Journal of Biosciences, 22(1). 48-52. Jan 2015.

[10] Shukla, S., Park, J., Kim, D.H., Hong, S.Y., Lee, J.S., Kim, M., "Total phenolic content, antioxidant, tyrosinase and $\alpha$-glucosidase inhibitory activities of water soluble extracts of noble starter culture Doenjang, a Korean fermented soybean sauce variety," Food Control, 59: 854-861. Jan 2016.

[11] Watanabe, H., "Biological efficacies of Miso," Journal of the Brewing Society of Japan, 105(11). 714-723. Nov 2010.

[12] Ito, A., Gotoh, T., \& Fujimoto, N., "Chemoprevention of cancers by miso and isoflavones," Journal of Toxicologic Pathology, 11(2). 79-84. Jun 1998.

[13] Yoshida, T., "Black Soybean Seed Coat Polyphenol and Pinitol (Next Generation of Biologically Active Substances in Soybean)," Nippon Shokuhin Kagaku Kogaku Kaishi, 60(9). 534-539. Dec 2013.

[14] Yoshikawa, H., Kuwashima, C., Kotaru, M., " $\alpha$-amylase inhibitors in the seeds of the genus Phaseolus and their characteristics," Research bulletin of Kyoto Koka Women's University, 47. 227-237. Dec 2009.

[15] Saito, Y., Nishi, S., Koaze, H., Hironaka, K., and Kojima, M., "Antioxidant and Inhibitory Activity on $\alpha$-Amylase and $\alpha$ Glucosidase in Legume Polyphenols," Journal of the Japanese Society for Food Science and Technology, 54(12). 563-567. Jan 2007.

[16] Acquaviva, R., Russo, A., Galvano, F., Galvano, G., Barcellona, M.L., Li Volti, G., and Vanella, A., "Cyanidin and cyanidin 3-O- $\beta$-D-glucoside as DNA cleavage protectors and antioxidants," Cell Biology and Toxicology, 19(4). 243-252. Aug 2003.

[17] Lin, W.H., Yang, H.W., Hsu, C.K., Jhan, H.K., Lo, D.Y., "Black Soybean Shows Protective Function against Carbon Tetrachloride-induced Liver Damage in Sprague-dawely Rats," Journal of Botanical Sciences, 5(1). 7-15. Dec 2016.

[18] Kanamoto, Y., Yamashita, Y., Nanba, F., Yoshida, T., Tsuda, T., Fukuda, I., Nakamura-Tsuruta, S., Ashida, H., “A Black Soybean Seed Coat Extract Prevents Obesity and Glucose Intolerance by Up-regulating Uncoupling Proteins and Down-regulating Inflammatory Cytokines in High-Fat Diet-Fed Mice," Journal of Agricultural and Food Chemistry, 59(16). 8985-8993. Aug 2011.

[19] Higashi, K., Fermentation and Brewing (1): Guidance on Production Line and Analysis of Miso- Soy Sauce, 3rd Edition, 61-90, Korin Co., Ltd., Tokyo, Mar 2008.
[20] Saito, Y., "Characterization and bio-synthesis regulation of polyphenol in legumes seed," Journal of the Japanese Society for Food Science and Technology, 53(7). 380-385. 2010.

[21] Ikeda, R., Ohta, N., and Watanabe, T., "Changes of Isoflavones at Various Stages of Fermentation in Defatted Soybeans," Journal of the Japanese Society for Food Science and Technology, 42(5). 322-327. 1995.

[22] Takahata, Y., Ohnishi-Kameyama, M., Furuta, S., Takahashi, M., and Suda, I., "Highly polymerized procyanidins in brown soybean seed coat with a high radical-scavenging activity,” Journal of Agricultural and Food Chemistry, 49(12). 5843-5847. 2001.

[23] Martins, S.I.F.S., Van Boeke, M.A.J.S., "Melanoidins extinctioin coefficient in the glucose/glycine Maillard reaction,” Food Chemistry 83(1). 135-142. Oct 2003.

[24] Jiang, C.Y., Ci, Z.H., Kojima, M., “Antioxidant Activity, $\alpha$-Glucosidase and Lipase Inhibitory Activity in Rice Miso with Kidney Bean," Journal of Food and Nutrition Research, 6(8). 504-508. Aug 2018.

[25] Fujimoto(Nakagawa), T., Hosokawa, C., \& Jo, M., "Inhibitory effects of traditional fermented soybean products on $\alpha$-glucosidase activity," Memoirs of the Faculty of Human Development University of Toyama, 11(2). 83-88. Dec 2017.

[26] Momose, A., Goto, N., Hayase, H., Gomyo, T., Miura, M., "Effects of Miso (Soybean Paste) on Postprandial Blood Sugar Levels," Journal of the Japanese Society for Food Science and Technology, 57(2). 63-69. Jan 2010.

[27] Ademiluyi, A.O., Oboh, G., Boligon, A.A., Athayde, M.L., "Effect of fermented soybean condiment supplemented diet on $\alpha$ amylase and $\alpha$-glucosidase activities in Streptozotocin-induced diabetic rats," Journal of Functional Foods, 9: 1-9, Jul 2014.

[28] Lee, J.H., Hwang, C.E., Son, K.S., Cho, K.M., "Comparisons of nutritional constituents in soybeans during solid state fermentation times and screening for their glucosidase enzymes and antioxidant properties," Food Chemistry, 272(30). 362-371. Jan 2019.

[29] Dai, C., M, H., He, R., Huang, L., Zhu, S., Ding, Q., Wo, L., "Improvement of nutritional value and bioactivity of soybean meal by solid-state fermentation with Bacillus subtilis," LWT, 86: 1-7. Dec 2017.

[30] Hwang, I.G., Kim, J.Y., Woo, K.S., Lee, J., and Jeong, H.S., "Biological activities of Maillard reaction products (MRPs) in a sugar-amino acid model system," Food Chemistry, 126(1). 221-227. 2011.

[31] Ademiluyi, A.O., Oboh, G., "Soybean phenolic-rich extracts inhibit key-enzymes linked to type 2 diabetes ( $\alpha$-amylase and $\alpha$-glucosidase) and hypertension (angiotensin I converting enzyme) in vitro," Experimental and Toxicologic Pathology, 65(3). 305-309. Mar 2013. 